\title{
IDENTIFICAÇÃO DE REGIÕES PLUVIOMÉTRICAS HOMOGÊNEAS NA BACIA HIDROGRÁFICA DO RIO PARANÁ
}

\author{
IDENTIFICATION OF HOMOGENEOUS RAINFALL REGIONS IN THE PARANÁ RIVER \\ HYDROGRAPHIC BASIN

\section{Maria Gabriela Louzada MALFATTI, Andréa de Oliveira CARDOSO, Diana Sarita HAMBURGER}

Universidade Federal do ABC, Centro de Engenharia, Modelagem e Ciências Sociais Aplicadas. Santo André, SP. Email: mglmalfatti@hotmail.com; andrea.cardoso@ufabc.edu.br; diana.sarita@ufabc.edu.br

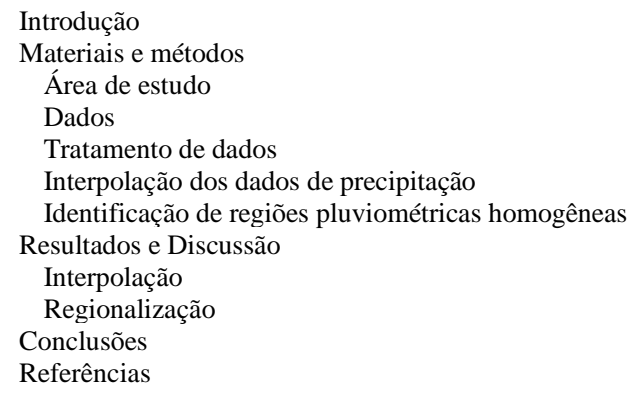

RESUMO - O objetivo deste trabalho foi identificar e analisar regiões pluviométricas homogêneas na bacia hidrográfica do Rio Paraná, por meio da técnica de análise de agrupamento (cluster). Foram utilizados dados diários de precipitação de 789 estações pluviométricas no período de 31 anos (1980 a 2010). Como a distribuição espacial dos dados é irregular, sua interpolação contribuiu para inferir valores em locais não amostrados. O método da Krigagem ordinária foi utilizado para este fim, sendo que o modelo exponencial se apresentou como o mais indicado, por possuir os menores erros estatísticos e maior dependência espacial, quando comparados aos modelos esférico e gaussiano. A partir desta análise, foi possível identificar grandes áreas que apresentam padrões semelhantes de precipitação na região de estudo. A interpolação dos dados auxiliou na definição mais detalhada de regiões homogêneas de precipitação, pela análise de cluster. Foram obtidas oito regiões com comportamentos pluviométricos distintos: as regiões ao sul da bacia apresentam menor variação ao longo dos meses do ano, com picos na primavera e outono, enquanto que as regiões ao norte e central da bacia apresentam um ciclo anual bem definido, caracterizado por uma estação seca no inverno e uma estação chuvosa no verão.

Palavras-chave: estações pluviométricas, regiões homogêneas, análise de agrupamento e interpolação de dados.

\begin{abstract}
The aim of this study was to identify and analyze homogeneous rainfall regions in the Parana River basin, through cluster analysis technique. It was used daily data of precipitation from 789 rainfall stations in a period of 31 year (1980-2010). As the spatial distribution of rainfall data is irregular, the data interpolation contributed in filling the data in locations not sampled. The method of ordinary kriging was used, and the exponential model is presented as the most suitable for presenting the smallest errors. From this analysis, it was possible to identify large groups that have similar patterns of rainfall in the study area. The interpolation of the data helped the more detailed definition of homogeneous regions of precipitation, by cluster analysis. Getting eight regions with different precipitation behavior: the southern regions of the basin, have less variation over the months of the year, with peaks in spring and fall, while the regions north and center of the basin, have a well-defined annual cycle, characterized by a dry season in winter and a rainy season in summer.
\end{abstract}

Keywords: precipitation stations, homogeneous regions, cluster analysis and data interpolation.

\section{INTRODUÇÃO}

O conhecimento da variabilidade da precipitação pluviométrica apresenta grande importância, visto que auxilia no planejamento e na tomada de decisão dos usos múltiplos da água, tais como, irrigação, consumo industrial, geração de energia e abastecimento público (Chierice \& Landim, 2014; Molina et al., 2014).

No planejamento e gerenciamento dos usos múltiplos da água utiliza-se a bacia hidrográfica como unidade territorial, onde são feitas as análises do comportamento do ciclo hidrológico da região em questão. A bacia hidrográfica é um sistema físico complexo, que possui diferentes propriedades físicas, heterogêneas e variáveis no tempo. Este sistema é representado matematicamente através de modelos, a fim de representar a dinâmica física real (Tucci, 2007).

O modelo mais simples em hidrologia é representado pela equação do balanço hídrico, sobre um determinado intervalo de tempo em uma bacia hidrográfica, onde a precipitação tem um papel fundamental na análise da disponibilidade hídrica da bacia (Tucci, 2007).

É conhecido que a precipitação é uma variável aleatória, influenciada por processos de diferentes escalas e que possui grande variabilidade espacial e temporal (Satyamurty et al., 1998; Grimm, 2003; Cavalcanti et al., 2009). Neste sentido, estudos de valores médios 
mensais e anuais de precipitação associados a distribuições de probabilidades permitem a avaliação dos riscos assumidos em projetos para o uso dos recursos hídricos (Cruz, 2009).

Uma característica usual das bases de dados pluviométricos em bacias brasileiras é a baixa e irregular distribuição espacial, principalmente em áreas distantes de grandes centros urbanos. Assim, algumas faixas do território brasileiro não possuem dados disponíveis, como ocorre em algumas partes da Bacia do Paraná, sendo, portanto, necessário aplicar técnicas de interpolação de dados, um método geoestatístico que infere sobre a precipitação em locais não amostrados.

$\mathrm{O}$ processo de interpolação de dados converte dados pontuais em uma grade com espaçamento regular. Existem vários métodos de interpolação de dados, alguns deles são: Krigagem (simples, ordinária, universal, indicação, disjunta e Co-krigagem), por Inverso da Distância (IQD), Thin Plate Spline (TPS), Radial Basin, Inverso da Potência da Distância (IPD), Inverso do Quadrado da Function Multiquadric (RBQ) (Loureiro \& Lisboa, 2011).

A interpolação de dados de precipitação foi considerada em diversos trabalhos. Viola et al. (2010) utilizaram métodos de interpolação para o mapeamento da precipitação pluvial em Minas Gerais, destacando que o método da Cokrigagem obteve o melhor resultado. Léllis \& Barroso (2011) interpolaram dados da precipitação na bacia do Rio Jacaraípe (Serra ES) através do método da Krigagem ordinária. Loureiro \& Lisboa (2011) compararam quatro métodos de interpolação de dados (Thin Plane Spline, Krigagem ordinária, IQD e RBQ) usando observações de precipitação na Bacia do Tocantins-Araguaia e concluíram que o método mais eficiente foi da Krigagem ordinária. Ávilla et al. (2009) utilizaram o método da Krigagem ordinária para o mapeamento da precipitação mínima provável para o sul de Minas Gerais. Barbosa (2006) também interpolou os dados para análise e espacialização de dados climáticos sobre o Estado de São Paulo pelo mesmo método. Mello et al. (2012) analisaram a distribuição de precipitação anual na bacia hidrográfica do Rio Cubatão Norte através do método da Krigagem ordinária.

Uma distribuição espacial regular dos dados auxilia na caracterização de regiões com comportamento similares, que podem ser encontradas a partir de técnicas de agrupamento de informações do regime e da variabilidade da precipitação pluviométrica. Na climatologia essa técnica é bastante utilizada para identificar áreas homogêneas de chuva. Por exemplo, Cardoso \& Silva Dias (2004) identificaram regiões com padrão homogêneo no Sul e Sudeste a partir do método de análise de agrupamento considerando as médias e limiares quantílicos sazonais de precipitação. O estudo de Barbosa (2006) identificou áreas homogêneas no Estado de São Paulo, com os dados de totais pluviométricos mensais e anuais. Souza et al. (2012) aplicou tal método sobre dados pluviométricos médios trimestrais e anuais sobre Mato Grosso do Sul e Oliveira (2014) caracterizou regiões no Nordeste do Brasil, a partir dos quantis de precipitação.

O objetivo deste trabalho é obter regiões homogêneas de precipitação, a partir dos dados pluviométricos, aplicando o método de interpolação de dados (Krigagem ordinária), visando obter uma distribuição regular dos dados de precipitação ao longo da Bacia do Paraná, e então, a partir desse conjunto de dados regulares aplicar uma análise de agrupamento para identificar regiões com comportamento homogêneo, o que permitirá a caracterização do regime pluviométrico ao longo da bacia.

\section{MATERIAIS E MÉTODOS}

\section{Área de estudo}

A região da Bacia Hidrográfica do Rio Paraná (BHRP) abrange as bacias hidrográficas do Paraná e Paraguai, inclui parte dos estados de São Paulo, Paraná, Mato Grosso do Sul, Mato Grosso, Minas Gerais, Goiás, Santa Catarina e Distrito Federal. O principal rio desta bacia é o Rio Paraná que nasce da comfluência dos rios Paranaíba e Grande, na divisa entre os estados de São Paulo, Minas Gerais e Mato Grosso do Sul, possuí uma extensão de $2.570 \mathrm{Km}$ até sua foz no Rio Prata, e seus principais afluentes são os rios Paranaíba, Grande, Tietê, Paranapanema e Iguaçu (ANA, 2009). A figura 1 ilustra a localização da área de estudo no Brasil, a divisão administrativa em sub-bacias da Agência Nacional de Águas (ANA) e sua hidrografia. 


\section{Dados}

Os dados de precipitação utilizados estão disponíveis no site da Agência Nacional das Águas (ANA). Este conjunto é composto por séries históricas de precipitação diária. O critério de seleção dos postos pluviométricos baseou-se no tamanho do período de registro e na sobreposição de estações ao longo da bacia, ou seja, buscou-se um conjunto de dados que agregasse um grande número de estações pluviométricas bem distribuídas espacialmente e um longo período de dados. Para tanto, determinou-se o período de estudo de 1980 a 2010, que resultou em um conjunto composto por 798 estações pluviométricas distribuídas sobre a BHRP, nos locais destacados na figura 2.

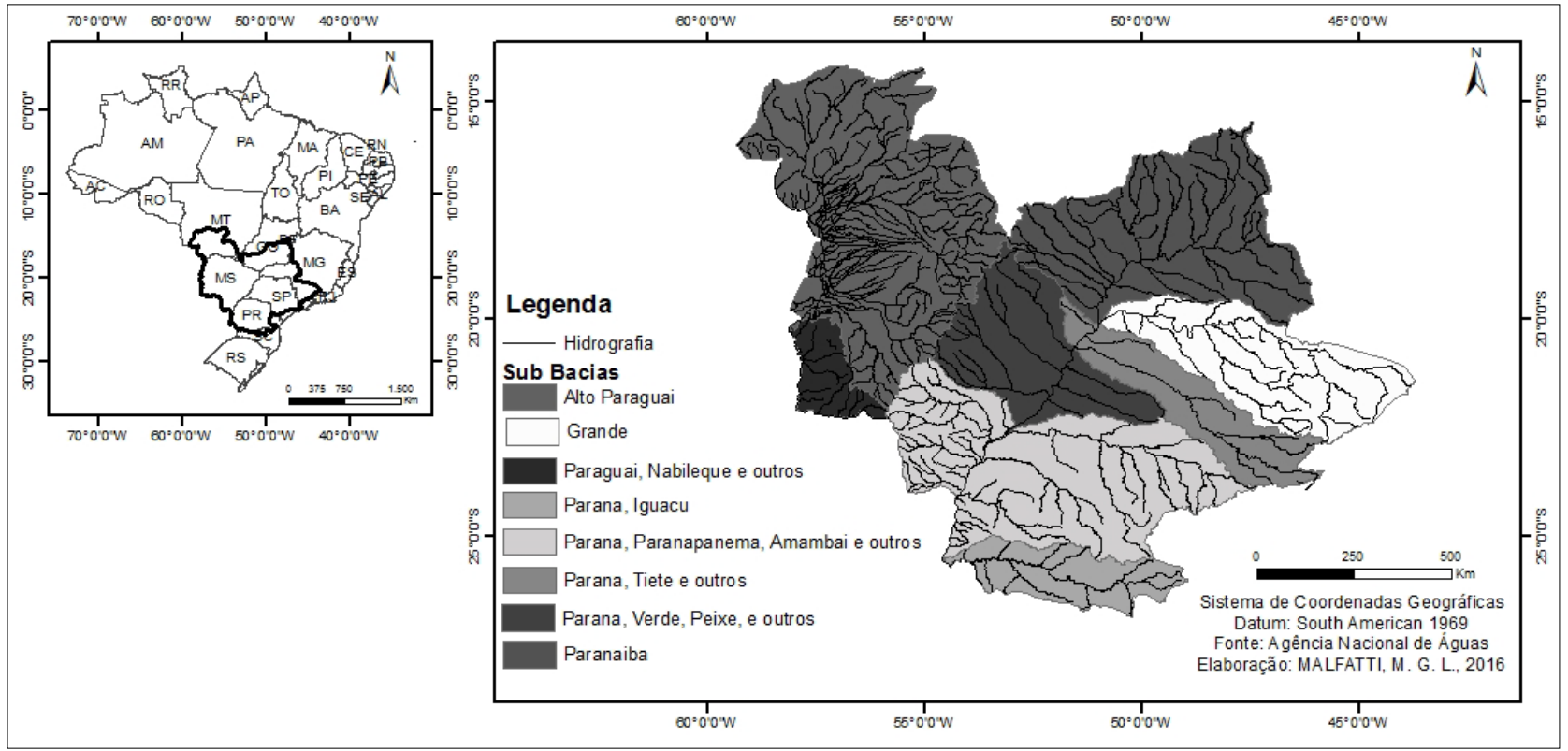

Figura 1 - Localização da Bacia Hidrográfica do Rio Paraná, seus principais rios e sub-bacias.

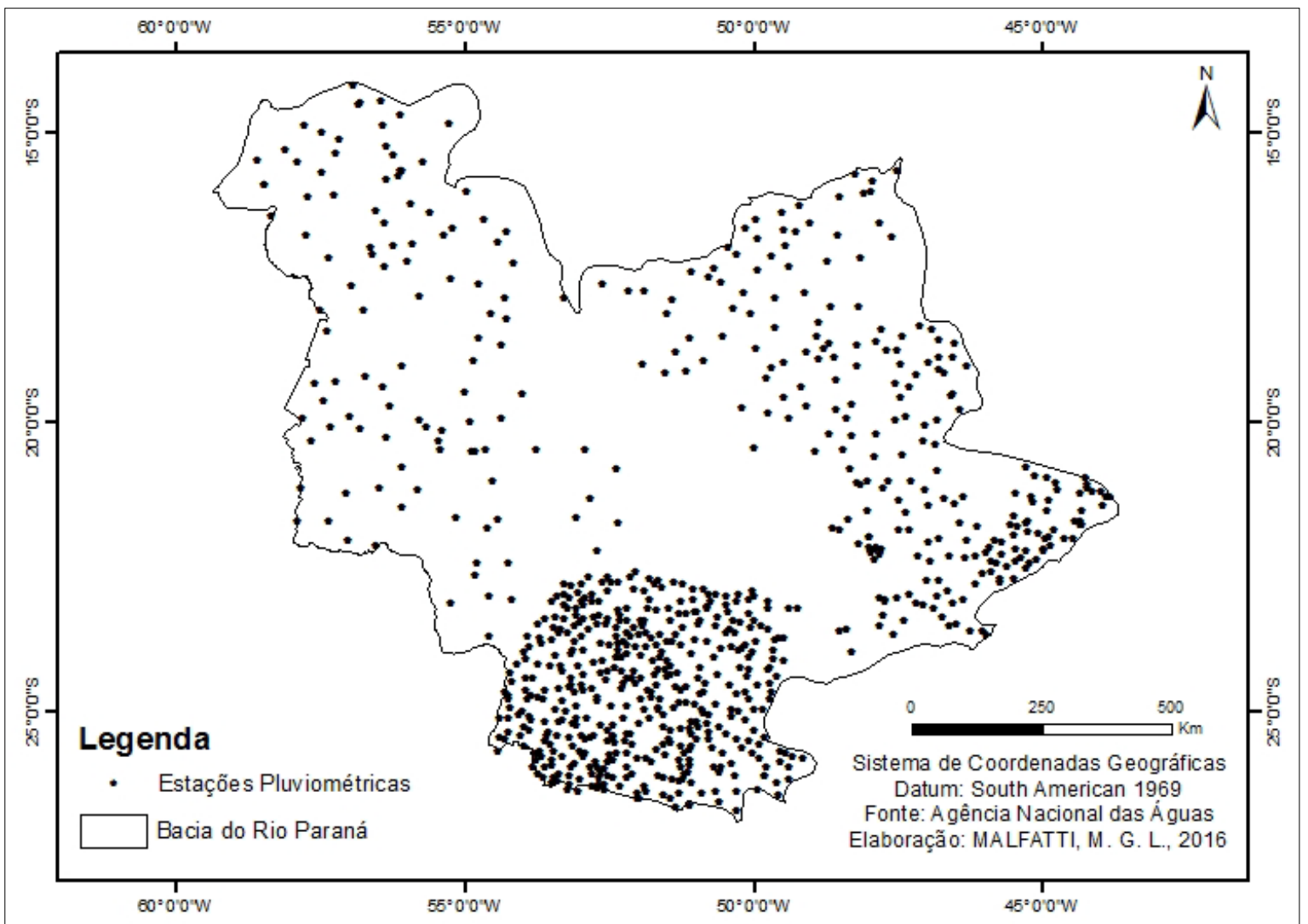

Figura 2 - Localização das estações pluviométricas na Bacia do Paraná. 


\section{Tratamento dos dados}

Para o desenvolvimento desse estudo foi necessário utilizar um conjunto de dados com séries históricas longas (mínimo 30 anos), contudo os dados de precipitação raramente possuem séries históricas longas e completas, frequentemente verificam-se falhas ao longo do conjunto de dados. No caso deste trabalho, como apenas $30 \%$ das séries originais são completas, foi necessário aplicar um método para preenchê-las.

O método de preenchimento de falhas consiste em estimar os dados faltantes através da técnica de regressão linear múltipla, sendo a estação a ser preenchida a variável dependente e as utilizadas no preenchimento as variáveis independentes (Tucci, 2002). Os postos utilizados no preenchimento devem apresentar alta correlação, proximidade geográfica e padrões climatológicos semelhantes. No caso deste trabalho foi realizada a seleção das estações vizinhas, através do coeficiente de correlação. Nos casos em que havia vizinhas com correlações superiores a 0,98 e dados no período de falha, aplicou-se uma regressão simples, utilizando a vizinha com série mais longa e mais próxima. No entanto, a condição anterior é mais rara, sendo que na maior parte dos casos o preenchimento foi realizado considerando as três estações vizinhas mais correlacionadas e com correlações superiores a 0,85 . O preenchimento foi realizado para cada mês separadamente, considerando anomalia de precipitação (a fim de evitar a ocorrência de séries altamente correlacionadas apenas pela influência do ciclo sazonal). Após o preenchimento da anomalia, o valor original é obtido inserindo o ciclo sazonal da própria estação preenchida.

Além disso, alguns dados podem apresentar valores muito discrepantes da maioria, chamados de dados outliers, decorrentes de erros de medição, erros de execução (falha do método empregado) ou da variabilidade inerente dos elementos (extremos) (Naghettini \& Pinto, 2007).

Em análises geoestatísticas, os outliers podem influenciar no comportamento dos semivariogramas experimentais na parte inicial, conduzindo à conclusão errada sobre a variância do ruído (efeito pepita) e ou a existência de correlação espacial na interpolação de dados (Ribeiro Júnior \& Diggle, 2001).
Para identificação de possíveis dados duvidosos foi utilizada a técnica do Diagrama de Box Plot sobre os dados médios de precipitação mensal acumulada. Este método consiste em um retângulo definido pelos primeiro e terceiro quartis da amostra ordenada (25\% e 75\%, respectivamente), contendo a mediana (50\%) em seu interior (Naghettini \& Pinto, 2007).

As estações identificadas com dados duvidosos causados por erros de registro foram excluídas da base de dados original (nove estações pluviométricas), resultando em 789 estações pluviométricas a serem consideradas no estudo. Estas estações pluviométricas estão localizadas ao sul da bacia, região com alta densidade de pontos, portanto as exclusões dessas estações não comprometeram as análises futuras.

Após o preenchimento dos dados, mais de $80 \%$ das estações pluviométricas estudadas passaram a ser completas, obtendo um registro mais confiável, visto que, anteriormente apenas $30 \%$ das séries de precipitação não possuíam dados faltantes.

\section{Interpolação dos dados de precipitação}

A interpolação dos dados de precipitação foi necessária para obter uma distribuição regular dos dados ao longo da bacia do Rio Paraná, principalmente em locais não amostrados. Para isso, utilizou-se uma grade regular de $1^{\circ}$ de latitude por $1^{\circ}$ de longitude, a fim de limitar a área que envolverá a superfície de ajuste.

Primeiramente os dados de precipitação foram categorizados, visando tornar a elaboração da interpolação mais clara e objetiva, comparando as diferentes estações de precipitação através de limiares representativos desta variável. Para tanto, foram calculados os valores da média e dos quantis de 15\%, 35\%, $65 \%$ e $85 \%$ da precipitação acumulada anual, visando identificar limiares que representem a dispersão da precipitação e, assim, caracterizem a amplitude da variabilidade temporal das séries.

Para calcular os quantis de uma determinada série de dados $\mathrm{P}_{\mathrm{i}}$, com $\mathrm{i}$ variando de 1 a $\mathrm{n}$ observações, é necessário determinar o número de ordem $\mathrm{w}\left(\mathrm{P}_{\mathrm{i}}\right)$ de cada elemento i desta série, admitindo que as observações estejam dispostas em ordem crescente. Sendo n o número de observações disponíveis, calculam-se os quantis a partir da (equação 1). 
$\mathrm{q}\left(\mathrm{P}_{\mathrm{i}}\right)=\frac{1}{2 \mathrm{n}}+\frac{\left[\mathrm{w}\left(\mathrm{P}_{\mathrm{i}}\right)-1\right]}{\mathrm{n}}$

Conforme o critério de Xavier \& Xavier (1987), os quantis são classificados como muito seco, se $\mathrm{P}_{\mathrm{i}} \leq 0,15$; seco, se $0,15<\mathrm{P}_{\mathrm{i}} \leq 0,35$; normal, se $0,35<\mathrm{P}_{\mathrm{i}} \leq 0,65$; chuvoso, se $0,65<\mathrm{P}_{\mathrm{i}}$ $\leq 0,85$ e muito chuvoso, se $P_{i}>0,85$.

Neste estudo utilizou-se o método geoestatístico de interpolação de dados chamado Krigagem ordinária, sendo este o mais conhecido e utilizado para interpolar dados de precipitação (Léllis \& Barroso, 2011). Este método se diferencia dos demais na definição dos pesos de ponderação obtidos através do ajuste dos modelos teóricos para estimar valores pluviométricos em locais não amostrados, obedecendo aos critérios de não-tendenciosidade e mínima variância (Viola et al., 2010).

Além disso, considera a dependência espacial que possibilita o controle de uma parcela do erro aleatório. Para isso, aplica-se a técnica de regressão que procura minimizar a variância estimada, através de um modelo de dependência estocástica entre os dados distribuídos no espaço, cujo valor esperado para o erro seja nulo e que apresentem variância mínima representada por uma função denominada variograma (Caruso \& Quarta, 1998).

A equação do estimador de Krigagem ordinária é definida a partir da combinação linear dos valores de um subconjunto amostral local (equação 2).

$\mathrm{z}\left(\mathrm{x}_{\mathrm{j}}, \mathrm{y}_{\mathrm{j}}\right)=\sum_{\mathrm{i}=1}^{\mathrm{n}(\mathrm{j})} \lambda_{1}\left(\mathrm{x}_{\mathrm{j}}, \mathrm{y}_{\mathrm{j}}\right) * \mathrm{z}\left(\mathrm{x}_{\mathrm{j}}, \mathrm{y}_{\mathrm{j}}\right)$

- onde z é o valor da precipitação a ser estimado; $\lambda_{1}$ é o peso associado ao estimador e $\left(\mathrm{x}_{\mathrm{j}}, \mathrm{y}_{\mathrm{j}}\right)$ são localizações espaciais sem informação.

A correlação espacial entre os pontos pode ser quantificada por meio do variograma experimental, expressando o comportamento espacial da variável contínua amostrando sua zona de influência, seus aspectos anisotrópicos e a presença de anomalias provocadas por erros de amostragem ou por componentes aleatórios.

O variograma é calculado como a média do quadrado da diferença entre os componentes dos pares de dados, porém na prática a correlação espacial entre os pontos é calculada a partir do semivariograma experimental (equação 3), ou seja, metade do valor do variograma (Goovaerts, 2000).

$\gamma(\mathrm{h})=\frac{1}{2 \mathrm{~N}(\mathrm{~h})} \sum_{\mathrm{i}=1}^{\mathrm{N}(\mathrm{h})}\left[\mathrm{Z}\left(\mathrm{S}_{\mathrm{i}}\right)-\mathrm{Z}\left(\mathrm{S}_{\mathrm{i}}+\mathrm{h}\right)\right]^{2}$

- onde h representa cada distância considerada entre quaisquer dois pontos; $\mathrm{Z}\left(\mathrm{S}_{\mathrm{i}}\right)$ é o valor da variável; $\mathrm{Z}$ medida em um local distanciado de S por uma distância $h$; $S_{i}$ é a posição amostral; $\mathrm{N}$ é o número de pares de pontos formados para uma dada distância $h$; $\gamma(\mathrm{h})$ é a semivariância estimada.

Após a obtenção dos semivariogramas experimentais foram testados os modelos: esférico (equação 4), exponencial (equação 5) e gaussiano (equação 6). O ajuste desses modelos é feito interativamente, manipulando-se os parâmetros $\left(a, C\right.$ e $C_{0}$ ) até que a função seja adequada (Goovaerts, 2000).

$$
\begin{aligned}
& \gamma_{1}(\mathrm{~h})=\left\{\begin{array}{l}
\mathrm{C}_{0}+\mathrm{C}_{1}\left[\frac{3 \mathrm{~h}}{2 a}-\frac{1}{2}\left(\frac{\mathrm{h}}{a}\right)^{3}\right], 0<h<a \\
\mathrm{C}_{0}+\mathrm{C}_{1}{ }^{3} \mathrm{~h} \geq a
\end{array}\right. \\
& \gamma_{2}(\mathrm{~h})=\mathrm{C}_{0}+\mathrm{C}_{1}\left\{1-\exp \left(-3 \frac{\mathrm{h}}{a}\right)\right\} \\
& \gamma_{3}(\mathrm{~h})=\mathrm{C}_{0}+\mathrm{C}_{1}\left\{1-\exp \left[-3\left(\frac{\mathrm{h}}{a}\right)^{2}\right]\right\}
\end{aligned}
$$

- onde $\mathrm{C}_{0}$ é o efeito pepita, ponto inicial da curva, onde a curva toca o eixo $\gamma$, quando $h=0$; $\mathrm{C}$ é o patamar, valor de $\gamma$ máximo da curva, o ponto em que não existe mais nenhuma correlação entre as variáveis, sendo assim a variância do conjunto de dados; $a$ é o alcance, o ponto máximo onde existe autocorrelação espacial das variáveis; $\mathrm{C}_{1}$ é a contribuição, diferença entre o patamar e o efeito pepita; e h é a distância entre dois pontos.

Para análise e escolha do modelo matemático que melhor se ajusta ao semivariograma experimental utilizou-se a técnica de validação cruzada (Isaaks \& Srivastava, 1989). Essa técnica consiste em retirar temporariamente do conjunto de dados um valor observado e estimá-lo a partir dos outros. Esse processo é feito para todos os valores observados, então são calculados os seguintes erros: Erro Viés (VIES) (equação 7), Raiz do Erro Quadrático Médio (REQM) (equação 8) e Erro Padrão Médio (EPM) (equação 9). 


$$
\begin{aligned}
& \text { VIES }=\frac{1}{n} \sum(F-0) \\
& \text { REQM }=\sqrt{\frac{1}{n} \sum(F-0)^{2}} \\
& \text { EPM }=\sqrt{\frac{1}{n} \sum \sigma^{2}}
\end{aligned}
$$

- onde, F é o valor modelado; O é o valor observado e $\sigma$ é o desvio padrão.

Após o ajuste dos modelos, foi obtido o grau de dependência espacial, através do índice de dependência espacial (IDE), expresso pela razão percentual entre o efeito pepita $\left(\mathrm{C}_{0}\right)$ e a contribuição $\left(\mathrm{C}_{1}\right)$ (equação (10).

$$
\mathrm{IDE}=\frac{\mathrm{C}_{0}}{\mathrm{C}_{1}+\mathrm{C}_{0}} \times 100 \%
$$

O IDE representa a porção da variabilidade espacial ao acaso, a partir dos critérios estabelecidos por Cambardella et al. (1994), onde a dependência espacial pode ser classificada em forte, se IDE $\leq 25 \%$; moderada, se $25 \%<$ IDE $<$ $75 \%$; e fraca, se IDE $>75 \%$.

O cálculo do semivariograma experimental, os ajustes dos modelos teóricos, a validação cruzada e a interpolação dos dados pluviométricos, foram realizados com o suporte da extensão Geostatistical Analyst presente no software ArcGIS 10.2.2 @ (ESRI).

\section{Identificação de regiões pluviométricas homogêneas}

Para agrupar as informações obtidas na interpolação de dados (média e dos limites quantílicos) foi utilizado o método de classificação não supervisionada por análise de agrupamento, função isocluster, disponível no software IDRISI ${ }^{\circledR}$ Taiga (Richards, 1993). Neste método o número de clusters é definido pelo usuário, no caso deste trabalho, inicialmente foram definidos doze clusters, duas vezes o número obtido por Cardoso (2005) (trabalho semelhante de regionalização para as regiões Sul e Sudeste do Brasil). Posteriormente, no desenvolvimento da regionalização foram reagrupadas pequenas sub-regiões, que apresentavam poucas estações contidas no interior de outra região maior ou na fronteira entre duas regiões, obtendo-se oito clusters.

A classificação não supervisionada, em processamento digital de dados espaciais, agrupa os dados, segundo as suas características, organizando-os em agrupamentos denominados de clusters. Nesta classificação alguns parâmetros são fornecidos pelo usuário como o número mínimo e máximo de classes desejadas e números de interações. No entanto as classes obtidas podem ou não coincidir com as classes de interesse, portanto, o procedimento é exploratório, verifica o que é estatisticamente separável ou não, quais as classes mais evidentes e sua relação com o que se deseja discriminar (Wilks, 2006).

O processo de classificação se inicia com um número aleatório de médias de agrupamentos uniformemente distribuídos no espaço de dados, representando uma configuração hipotética de centros de agrupamento. Assim, iterativamente, as distâncias mínimas são calculadas para cada elemento da grade e eles são reunidos nos agrupamentos mais próximos usando a técnica de distância mínima (menor distância ao centro dos agrupamentos) (Meneses \& Almeida, 2012).

Neste caso a distância mínima utilizada é a menor medida de distância Euclidiana, sendo que cada ponto da grade se incorpora a um agrupamento através da análise da medida de similaridade de distância Euclidiana (equação 11).

$\mathrm{D}(\mathrm{x}, \mathrm{n})=\sqrt{\left(\mathrm{x}_{\mathrm{i}}-\mathrm{m}_{\mathrm{i}}\right)^{2}}$

- onde $\mathrm{D}(\mathrm{x}, \mathrm{n})$ é a distância euclidiana; $\mathrm{x}_{\mathrm{i}}$ é o ponto candidato; e $\mathrm{m}_{\mathrm{i}}$ é a média das classes.

No término dessa sequência de alocação, a média dos valores de todos os elementos da grade alocados a cada agrupamento arbitrário é computada para cada uma das variáveis, obtendose as coordenadas dos centros de cada cluster.

A partir desses centros uma nova iteração é realizada e a cada nova iteração é recalculada a média, e os valores são então reclassificados com relação às novas médias (Meneses \& Almeida, 2012).

Após a escolha das regiões homogêneas foram determinadas séries históricas de precipitação média por região, para os anos de 1980 a 2010, considerando-se as estações pluviométricas de cada grupo resultante da análise de agrupamento. Posteriormente, foram calculados os valores da média e dos limiares quantílicos com base no histórico de cada mês do ano. 


\section{RESULTADOS E DISCUSSÃO}

\section{Interpolação}

Os resultados da validação dos modelos de semivariogramas exponencial, esférico e gaussiano, a partir da técnica de validação cruzada (VIES, REQM e EMP) e índice de dependência espacial (IDE) estão disponíveis na Tabela 1.

Para que o modelo de semivariograma seja considerado de melhor ajuste na interpolação dos dados, os erros do prognóstico devem ser contemplados de maneira integrada. De acordo com a Tabela 1, os valores do VIES indicam que o modelo exponencial apresenta menor erro sistemático para todos os limiares de precipitação analisados, além disso, os valores de EMP também foram mais baixos para este modelo. Os modelos esférico e gaussiano também apresentaram bons resultados para alguns limiares, destacando-se o REQM para o gaussiano e EMP para o esférico. Contudo, de um modo geral foi possível observar que o modelo exponencial apresenta menores valores em mais limiares, sendo considerado, a partir deste critério, o melhor modelo para a interpolação dos dados de precipitação na bacia estudada.

Tabela 1 - Acurácia dos modelos teóricos de semivariograma em mm.

\begin{tabular}{c|c|c|c|c|c}
\hline \multirow{2}{*}{ Limiares } & Modelo de & \multicolumn{4}{|c}{ Erros do Modelo } \\
\cline { 2 - 5 } & semivariograma & VIES & REQM & EMP & IDE (\%) \\
\hline \multirow{3}{*}{ Média } & Exponencial & $\mathbf{- 0 , 1 5}$ & 144,60 & $\mathbf{1 2 3 , 0 2}$ & $\mathbf{4 , 8 6}$ \\
\cline { 2 - 5 } & Esférico & $-0,26$ & 139,75 & 131,08 & 12,32 \\
\cline { 2 - 5 } & Gaussiano & $-0,46$ & $\mathbf{1 3 8 , 3 2}$ & 132,83 & 19,65 \\
\hline \multirow{3}{*}{ Quantil 15 } & Exponencial & $\mathbf{- 0 , 0 8}$ & 124,06 & 107,70 & $\mathbf{9 , 0 1}$ \\
\cline { 2 - 5 } & Esférico & $-0,19$ & 121,49 & $\mathbf{1 0 6 , 2 5}$ & 13,57 \\
\cline { 2 - 5 } & Gaussiano & $-0,56$ & $\mathbf{1 2 0 , 3 7}$ & 111,64 & 23,10 \\
\hline \multirow{3}{*}{ Quantil 35 } & Exponencial & $\mathbf{- 0 , 4 5}$ & 115,82 & 83,73 & 1,29 \\
\cline { 2 - 5 } & Esférico & $-0,50$ & 118,56 & $\mathbf{6 9 , 6 1}$ & $\mathbf{0 , 0 0}$ \\
\cline { 2 - 5 } & Gaussiano & $-0,51$ & $\mathbf{1 0 7 , 3 6}$ & 101,87 & 15,19 \\
\hline & Exponencial & $\mathbf{- 0 , 2 0}$ & 127,50 & $\mathbf{9 9 , 3 4}$ & $\mathbf{0 , 0 0}$ \\
\cline { 2 - 5 } & Esférico & $-0,21$ & 120,40 & 106,85 & 6,55 \\
\cline { 2 - 5 } & Gaussiano & $-0,28$ & $\mathbf{1 1 8 , 8 6}$ & 109,44 & 13,88 \\
\hline \multirow{3}{*}{ Quantil 85 } & Exponencial & $\mathbf{0 , 1 0}$ & 162,15 & $\mathbf{1 3 5 , 0 5}$ & $\mathbf{4 , 3 3}$ \\
\cline { 2 - 5 } & Esférico & $-0,18$ & 157,30 & 144,63 & 11,69 \\
\cline { 2 - 5 } & Gaussiano & $-0,45$ & $\mathbf{1 5 6 , 1 6}$ & 146,65 & 18,81 \\
\hline
\end{tabular}

Segundo o cálculo do índice de dependência, todos os modelos de semivariograma considerados no estudo apresentam IDE $\leq$ $25 \%$, indicando forte dependência espacial. No entanto, o modelo de semivariograma teórico exponencial apresenta os menores IDE apresentando uma dependência espacial maior do que os outros modelos teóricos. Desta forma, o modelo que apresentou o melhor ajuste foi o modelo exponencial, sendo, portanto, o considerado na interpolação de precipitação no presente estudo.

Os resultados obtidos das interpolações de dados médios, quantis 15\%, 35\%, 65\% e 85\%, de precipitação acumulada anual, através do método da Krigagem ordinária, pelo modelo de semivariograma exponencial, estão representados na figura 3.

Em termos espaciais, verificou-se que os três modelos de ajuste apresentaram elevados valores de erro médio padrão (valores acima de $100 \mathrm{~mm}$ ), principalmente na região central da bacia, sendo provavelmente derivados da baixa distribuição de dados pluviométricos, ocasionando uma maior incerteza das interpolações nestas faixas.

Através da análise do erro médio padrão (EMP), disponível na figura 4, observa-se que os maiores erros estão concentrados na região central da BHRP e os menores erros na região sul da BHRP, demostrando que quanto menor (maior) a densidade de estações, maior (menor) será o erro do modelo de semivariograma.

Para os limites quantílicos o mesmo comportamento é verificado, quanto maior (menor) a densidade dos dados pluviométricos, menor (maior) será o erro do modelo de semivariograma teórico exponencial. 


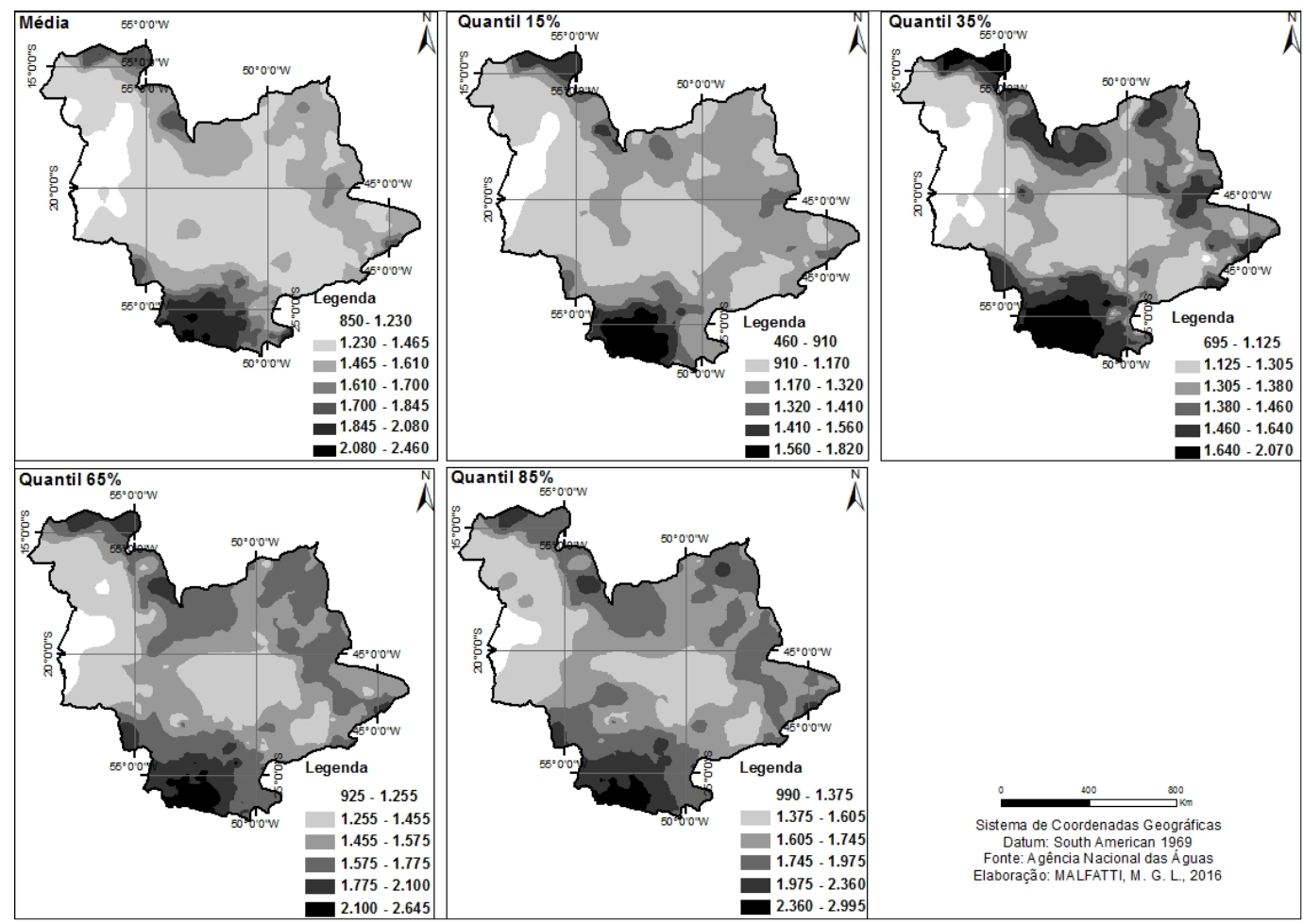

Figura 3 - Interpolação de dados pelo método da Krigagem, para o modelo de semivariograma exponencial, com as precipitações médias, quantis 15, 35, 65 e 85 acumuladas (mm).

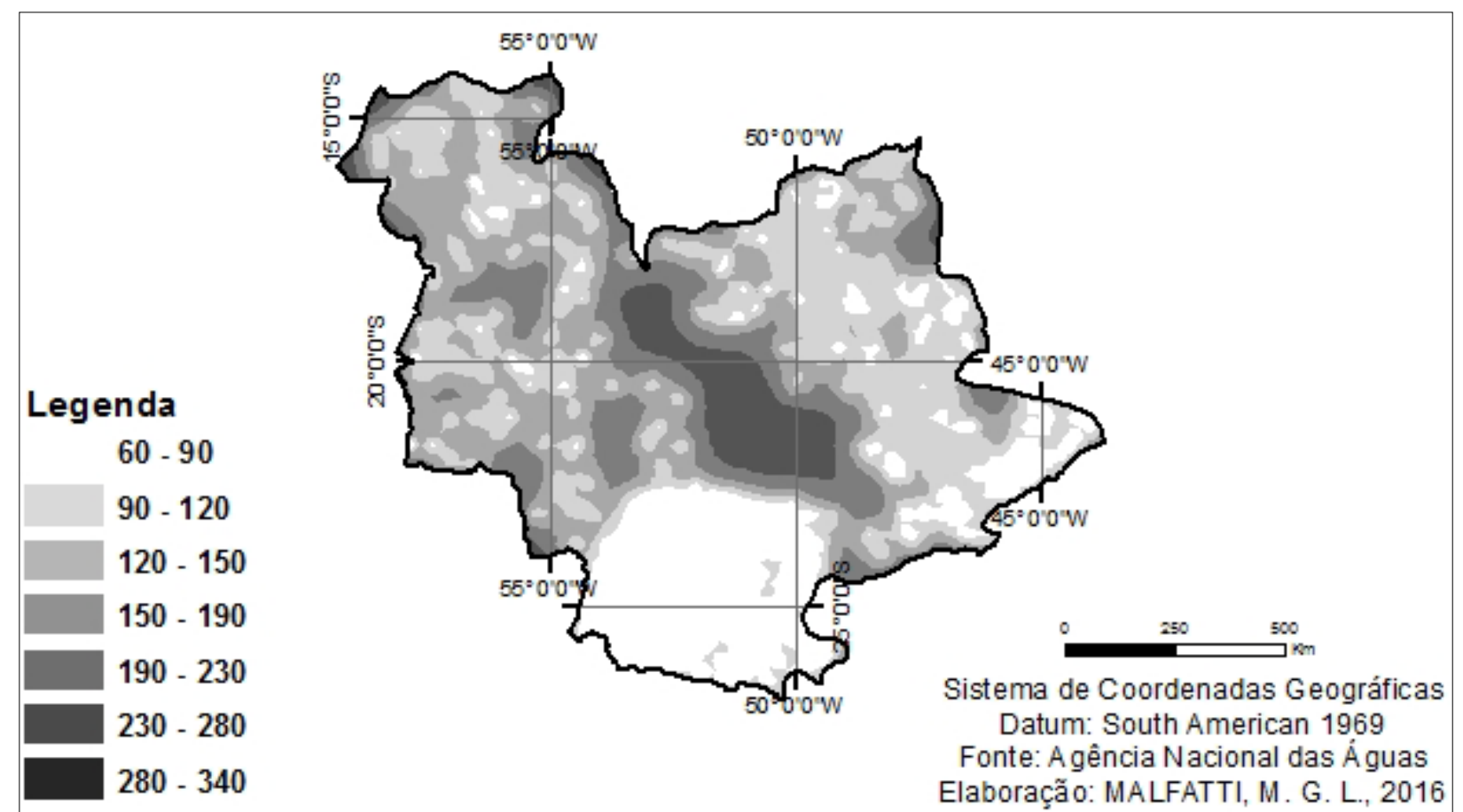

Figura 4 - EMP da interpolação de dados pelo método da Krigagem para o modelo exponencial de semivariograma para os dados médios de precipitação acumulada (mm).

A partir da interpolação dos dados pluviométricos foram identificadas faixas com características de precipitação semelhantes na área de estudo, tais como: Região Sul da Bacia (estado do PR, sul de SC e sul de SP) Região Central (estado de SP), Região ao Leste (sul de MG, sul de GO e leste de SP), Região Noroeste (MT) e Região Oeste (sul de MS) corroborando com os resultados obtidos por Cardoso \& Silva Dias (2004), o qual realiza a regionalização do Sul e Sudeste do Brasil, regiões que abrangem grande parte da BHRP.

\section{Regionalização}

Na figura 5 observa-se o resultado obtido da análise de agrupamento (cluster) aplicada aos dados pluviométricos interpolados. A partir da 
técnica utilizada foram determinadas oito regiões pluviométricas homogêneas na BHRP.

No desenvolvimento da regionalização foram reagrupadas pequenas sub-regiões, que apresentavam poucas estações contidas no interior de outra região maior ou na fronteira entre duas regiões.

Nestes casos, tais estações foram englobadas em regiões maiores que apresentavam padrões similares, o que ocorreu principalmente nas regiões 1 e 4 .
A partir da figura 5 nota-se que a região 6 apresenta maior área, possivelmente em virtude da baixa densidade de estações pluviométricas na faixa central da bacia, levando a uma interpolação de dados com maior incerteza e consequentemente uma análise de agrupamento mais generalizada.

A região 5 apresenta menor área, por ser uma pequena região litorânea, caracterizada por regime e variabilidade bastante distintos em relação às regiões adjacentes 4 e 6 .

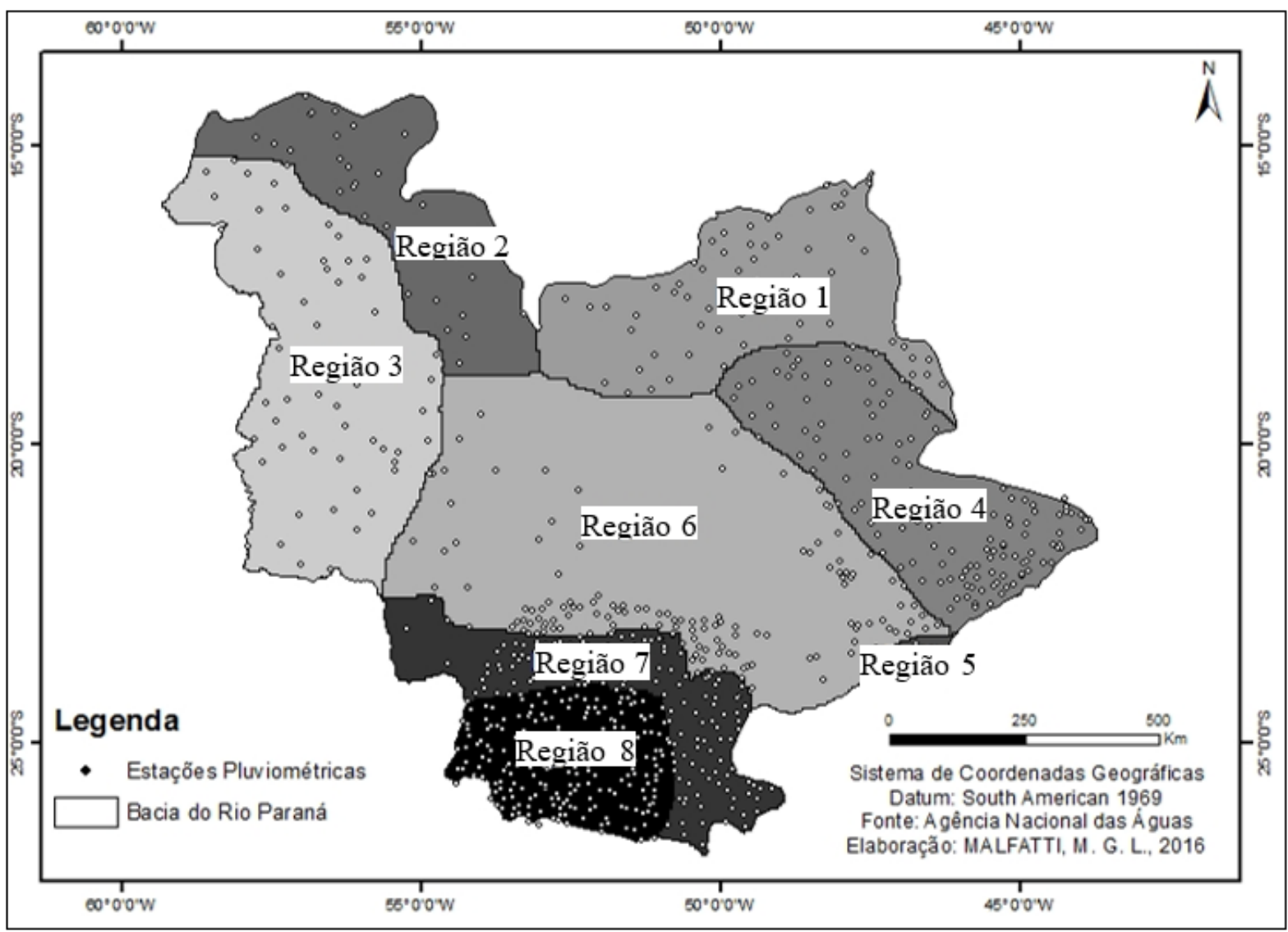

Figura 5 - Regionalização da precipitação na Bacia do Paraná para todas as estações do ano.

Após a identificação dos grupos com comportamento pluviométrico homogêneo, figura 5, calculou-se as precipitações acumuladas mensais e anuais médias sobre cada região homogênea. Posteriormente, transformou-se a precipitação em quantis, identificando os limiares das categorias de chuva para estas regiões no período de 1980 a 2010, visando caracterizar os padrões de precipitação distinguindo pela análise de cluster.

Na Tabela 2 se encontram os valores médios e quantílicos anuais para as regiões pluviométricas homogêneas. Por meio desta tabela observam-se, em ordem crescente, as regiões mais secas e mais chuvosas, de acordo com os acumulados anuais, cuja localização está expressa na figura 5 com tons cinza mais escuros representando as regiões mais chuvosas. Observa-se que as regiões ao sul e sudeste da bacia são as mais chuvosas (regiões 5,7 e 8), enquanto que as regiões mais ao norte (1 a 4) e central (6) são mais secas.

Essas diferenças são observadas devido a sistemas atuantes distintos na Bacia do Paraná. Segundo Reboita (2010) a região sul do Brasil, que no presente trabalho inclui as regiões 7 e 8 da BHRP, apresenta uma distribuição regular de chuvas anual. Alguns fenômenos atmosféricos que atuam sobre essa região são 
responsáveis por grande parte dos totais pluviométricos, o principal deles são os sistemas frontais. Além disso, ciclones e frentes frias que se desenvolvem devido à presença de vórtices ciclônicos ou cavados em altos níveis sobre a costa oeste da América do Sul, vindos do Pacífico, e também devido a condições frontogenéticas e/ou ciclogenéticas originadas na região.

$\mathrm{Na}$ faixa que engloba parte do sudeste e centro-oeste do Brasil (Regiões de 1 a 6, deste trabalho) existe um ciclo anual bem definido, com um período seco (inverno) e outro chuvoso (verão), caracterizado pelo Sistema de Monção da América do Sul (SMAS) (Grimm, 2005). Os mesmos sistemas que afetam a região Sul, podem atingir o Sudeste e sul do Centro-oeste, porém em menor frequência.

De acordo com os dados da tabela 2, nota-se que as regiões mais chuvosas apresentam maior variabilidade interanual e assimetria na distribuição das chuvas, sendo que os eventos considerados muito chuvosos $\left(\mathrm{P}_{\mathrm{i}}>0,85\right)$ apresentam diferenças mais expressivas, em termos dos totais pluviométricos, em relação aos eventos chuvosos $\left(0,65<\mathrm{P}_{\mathrm{i}} \leq 0,85\right)$, o que não se observa entre as demais categorias de precipitação. Destaca-se que as regiões 3 e 6 , que são menos chuvosas, apresentam menor variabilidade interanual e uma distribuição mais simétrica da precipitação.

Tabela 1 - Valores da precipitação anual (mm) relacionados a média, quantil 15\%, 35\%, 65\% e 85\% para as regiões pluviométricas homogêneas no período de 1980 a 2010.

\begin{tabular}{c|c|c|c|c|c|c|c|c}
\hline \multirow{2}{*}{ Dados } & \multicolumn{7}{c}{ Regiões } \\
\cline { 2 - 9 } & REG3 & REG6 & REG1 & REG4 & REG2 & REG5 & REG7 & REG8 \\
\hline Quantil 15\% & 945,66 & 1143,10 & 1190,98 & 1235,67 & 1233,69 & 1282,76 & 1271,26 & 1536,15 \\
\hline Quantil 35\% & 1100,59 & 1285,60 & 1380,80 & 1392,53 & 1415,84 & 1484,10 & 1439,47 & 1724,66 \\
\hline Média & 1220,44 & 1394,38 & 1480,76 & 1513,37 & 1528,76 & 1553,22 & 1569,11 & 1898,31 \\
\hline Quantil 65\% & 1301,04 & 1475,56 & 1585,46 & 1583,63 & 1629,87 & 1658,67 & 1646,54 & 1968,82 \\
\hline Quantil 85\% & 1459,00 & 1642,70 & 1757,93 & 1768,50 & 1811,33 & 1827,06 & 1851,48 & 2190,20 \\
\hline
\end{tabular}

A figura 6 mostra os valores de precipitação mensal média e os quantis de 15\%, 35\%, 65\% e $85 \%$, identificados para cada mês do ano, sobre as oito regiões pluviométricas homogêneas. Através desta figura é possível identificar os diferentes padrões de precipitação existentes ao longo da BHRP.

As regiões 7 e 8, sul da bacia, apresentam menor variação sazonal, com picos na primavera e no outono, principalmente para os limiares muito chuvosos (quantil 0,85), enquanto que as demais regiões ( 1 a 6 ), norte e centro da bacia, possuem um ciclo anual bem definido, caracterizado por uma estação seca no inverno (junho, julho e agosto) e uma estação chuvosa no verão (dezembro, janeiro e fevereiro).

As regiões 7 e 8 se diferenciam pela amplitude da precipitação, sendo que a região 8 apresenta limiar de $85 \%$ com valor máximo de $320 \mathrm{~mm}$ no mês de outubro, enquanto que para a região 7 o limiar de $85 \%$ apresenta valor máximo de $280 \mathrm{~mm}$ no mês de janeiro.

As regiões 1 a 6 possuem um ciclo anual caracterizado por uma estação seca e chuvosa, que se diferem pela amplitude da diferença entre máximos e mínimos, o que é verificado em todas as categorias de precipitação. As regiões 2, 3 e 4 (oeste e leste da bacia) apresentam um ciclo mais marcado, com estações secas bastante pronunciadas nos meses de inverno (junho, julho e agosto), período de menor variabilidade da precipitação. Em contrapartida, verifica-se que no período chuvoso há grande variabilidade da precipitação nestas regiões. Nas demais regiões (1, 5 e 6), faixa norte e central, a estação seca apresenta valores mais elevados e maior variabilidade.

Além disso, através dos limiares de precipitação nota-se que as regiões 2,3 e 4 apresentam valores muito próximos ao longo do ano, a diferença entre os limiares são observadas apenas em parte dos meses chuvosos.

Enquanto que para as outras regiões a diferença entre os limiares são expressas em todas as épocas do ano, com maior intensidade para as regiões 7 e 8 ao sul da BHRP, onde as chuvas são mais uniformes ao longo do ano.

Como foram considerados os anos de 1980 a 2010 para analisar o regime de precipitação da 
BHRP, os anos de 1982/83, 1987/1988, 1991/1993, 2002/2003 foram anos de El Niño, que influenciou na ocorrência de elevadas anomalias positivas de precipitação nas regiões Sul e sul do Sudeste do Brasil (regiões 6, 7 e 8 da BHRP) nos meses de outono, principalmente no mês de maio (média de $400 \mathrm{~mm}$, anomalias positivas maiores que $200 \mathrm{~mm}$ ) e também na primavera (Kousky et al., 1984; Cavalcanti, 1992; Minuzzi, 2006).

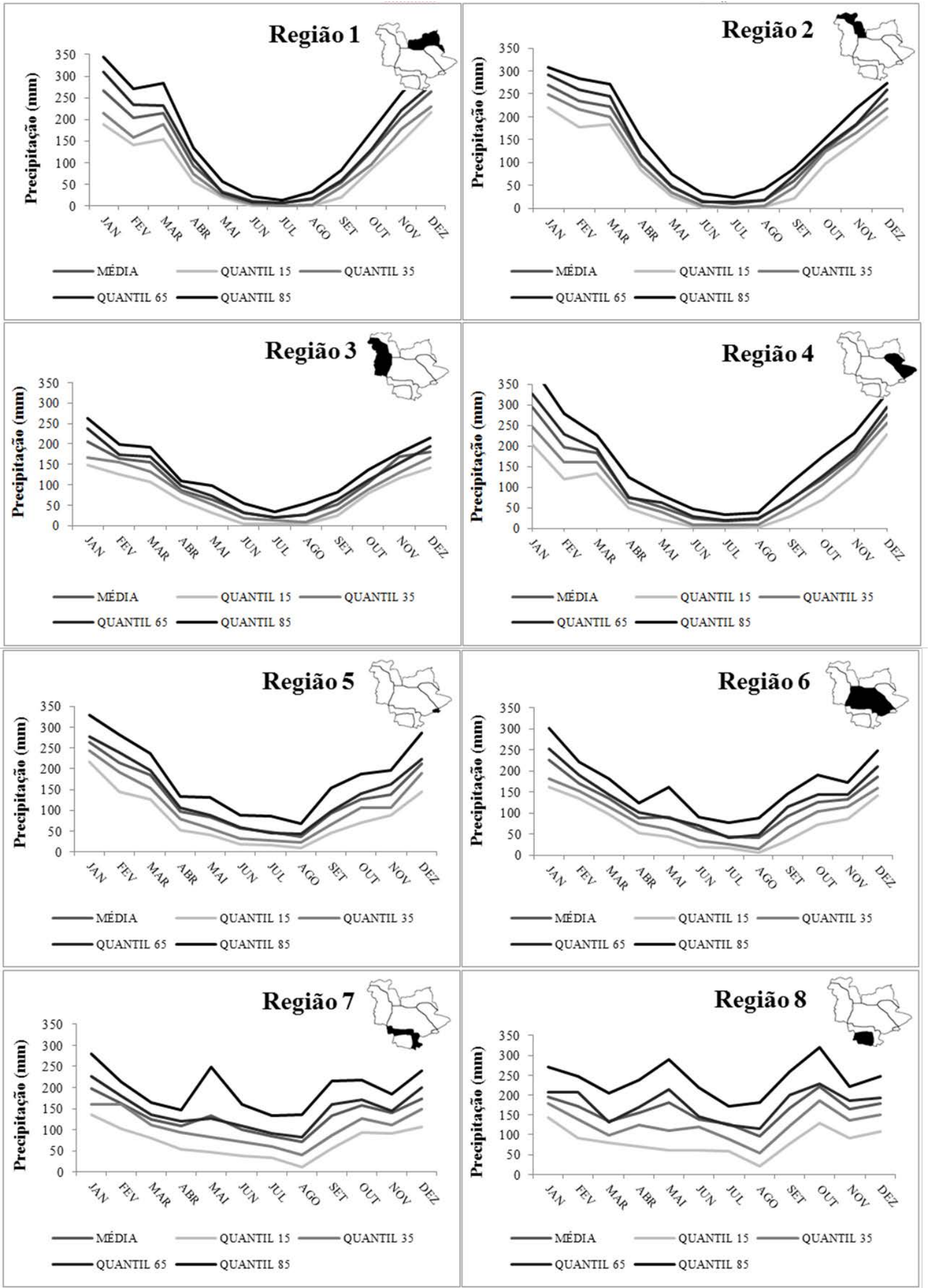

Figura 6 - Valores da precipitação mensal relacionados à média e aos quantis de 15\%, 35\%, 65\% e 85\%, calculados sobre cada região pluviométrica homogênea no período de 1980 a 2010. 


\section{CONCLUSÕES}

A aplicação de um método de interpolação sobre dados irregulares espacialmente, permite a obtenção de uma base de dados distribuídos regularmente, que facilita a identificação de faixas de precipitação com padrões semelhantes e a inferência sobre a precipitação nos locais não amostrados, tornando a análise de cluster mais confiável.

No então, é importante avaliar o ajuste na interpolação dos dados por diferentes modelos, visando identificar o mais adequado para o conjunto de dados específico.

O modelo exponencial utilizado no método da Krigagem ordinária apresentou os menores erros, para interpolar os dados de precipitação sobre a BHRP. A partir desta análise foi possível identificar grandes faixas que apresentam padrões semelhantes de precipitação dentro da área de estudo, como: Região Sul da Bacia, Região Central, Região ao Leste e Região Noroeste.

O agrupamento dos limiares quantílicos de precipitação anual, pelo método de cluster, gerou grupos de precipitações com distribuições homogêneas, levando em conta além do padrão médio, diferentes categorias de precipitação, o que torna a classificação mais criteriosa.

A partir desta técnica foram determinadas regiões pluviométricas distribuídas sobre a BHRP, que podem ser consideradas com regime homogêneo de precipitação, dada a métrica aplicada. Desta caracterização foram encontradas oito regiões com padrões distintos, sendo que as regiões ao sul da bacia apresentam menor variação ao longo dos meses do ano, com picos na primavera e outono, enquanto que as regiões ao norte e centro da bacia apresentam um ciclo anual bem definido, caracterizado por uma estação seca no inverno (junho, julho e agosto) e chuvosa no verão (dezembro, janeiro e fevereiro).

Além disso, observam-se diferenças entre os limiares quantílicos mensais, ao passo que nas regiões mais ao norte os limiares são mais distintos apenas em parte da estação chuvosa, enquanto que nas demais regiões as diferenças entre os limiares quantílicos são significativas ao longo do ano, com maior intensidade para as regiões ao sul da bacia.

\section{AGRADECIMENTOS}

Os autores agradecem à UFABC pelo apoio técnico e financeiro, à CAPES pela bolsa de mestrado do primeiro autor e ao CNPq, pelo apoio ao projeto Universal ( $\left.n^{\circ} 471700 / 2013-4\right)$.

\section{REFERÊNCIAS}

ANA - Agência Nacional de Águas (Brasil). Relatório de Conjuntura dos Recursos Hídricos do Brasil. Brasília, 2009. $204 \mathrm{p}$.

ÁVILLA, L.F.; MELLO, C.R.; VIOLA, M.R. Mapeamento da precipitação mínima provável para o sul de Minas Gerais. Revista Brasileira Engenharia Agrícola Ambiental, v. 13, suplemento, p. 906-915, 2009.

BARBOSA, J.P.M. Utilização de métodos de interpolação para análise e espacialização de dados climáticos: o SIG como ferramenta. Caminhos de Geografia, v. 9, n. 17, p.85-96, 2006.

CAMBARDELLA, C.A.; NOVAK, J.; MOORMAN, T.B.; KONOPKA, A. Field-scale variability of soil properties in central Iowa soils. Soil Science Society of America Journal, v. 58, n. 5, p. 1240-1248, 1994.

CARDOSO, A.O. \& SILVA DIAS, P.L. Identificação de trimestres extremos no regime pluviométrico do Sul e Sudeste do Brasil e relação com anomalias da TSM. Revista Brasileira de Meteorologia, v. 19, n. 2, p. 149-162, 2004.

CARDOSO, A.O. Relações entre a TSM nos oceanos Atlântico e Pacífico e as condições climáticas nas regiões Sul e Sudeste do Brasil. 2005. 192 f. Tese (Doutorado de Meteorologia) Instituto de Astronomia Geofísica e Ciências Atmosféricas, Universidade São Paulo, 2005.

CARUSO, C. \& QUARTA, F. Interpolation methods comparison. Computers \& Mathematics with Applications, v. 35, n. 12, p. 109-126, 1998.

CAVALCANTI, I.F.A. Episódios El Niño/Oscilação Sul durante a década de 1986 a 1996 e suas influências sobre o Brasil. In: CLIMANÁLISE ESPECIAL: EDIÇÃO COMEMORATIVA DE 10 ANOS. Atas...São José dos Campos: INPE/CPTEC, SP, p. 52-64, 1996.

CAVALCANTI, I.F.A.; FERREIRA, N.J.; SILVA, M.G.A.J.; MARIA ASSUNÇÃO FAUS DA SILVA DIAS, M.A.F.S. Tempo e Clima no Brasil. 1. ed. São Paulo: Oficina de Textos, 464p., 2009

CHIERICE, R.A.F. \& LANDIM, P.M.B. Variabilidade espacial e temporal de precipitação pluviométrica na bacia hidrográfica do Rio Mogi Guaçu. Geociência, v. 33, n. 1, p. 157-171, 2014.

CRUZ, M.A.S. Regionalização de precipitação médias e prováveis mensais e anuais no estado de Sergipe. Boletim de Pesquisa EMBRAPA, 2009.

GOOVAERTS, P. Geostatistical approaches for incorporating elevation into the spatial interpolation of rainfall. Journal of Hydrology, v. 228, n. 1-2, p. 113-129, 2000.

GRIMM, A.M. The El Nino impact on the summer monsoon in Brazil: Regional processes versus remote influences. Journal of Climate, v. 16, n. 2, p. 263-280, 2003.

GRIMM A.M.; VERA, C.; MECHOSO, C.R. The South American Monsoon System. The global monsoon system: research and forecast, World Meteorological Organization. Eds. Chang CP, Wang B, Lau NCG, WMO/TD, n. 1266, p.219-238, 2005.

ISAAKS, E.H. \& SRIVASTAVA, R.M. An Introduction to Applied Geostatistics. New York: Oxford University Press, 1989. 
KOUSKY, V.E.; KAYANO, M.T.; CAVALCANTI, I.F.A. A review of the southern oscillation oceanic atmospheric circulation changes and related rainfall anomalies. Tellus, 36 A: p. 490-504, 1984.

LÉLLIS, F.S. \& BARROSO, G.F. Modelagem geoestatística da precipitação pluviométrica na bacia do rio Jacaraípe, Serra (ES). In: Simpósio Brasileiro de Sensoriamento Remoto, 15, 2011, Curitiba. Anais... São José dos Campos: INPE, 2011.

LOUREIRO, G.E. \& LISBOA, E.G. Comparação dos métodos de Interpolação Espacial aplicados a dados pluviométricos da Região Hidrográfica Tocantins-Araguaia. In: Simpósio Brasileiro de Recursos Hídricos, 19, 2011, Maceió, Anais... Porto Alegre: ABRH, 2011.

MELLO, Y.R.; OLIVEIRA T.M.N.; VAZ, C. Distribuição de precipitação anual na bacia hidrográfica do rio Cubatão Norte. Revista Geonorte, Manaus, Ed. Especial 2, v. 1, n. 5, p. 535546, 2012.

MENESES, P.R. \& ALMEIDA, T. (Coords). Introdução ao processamento de imagens de sensoriamento remoto. Brasília: CNPq, 266p., 2012.

MINUZZI, R.B.; SEDIYAMA, G.C.; COSTA, J.M.N.; VIANELLO, R.L. Influência do fenômeno climático El Niño no período chuvoso da região sudeste do Brasil. Geografia, Londrina, v. 15, n. 2, p. 5-19, 2006.

MOLINA, S.K.M.; ALMEIDA, I.K.; STEFFEN, J.L.; ALVES SOBRINHO, T. Caracterização de disponibilidade hídrica e comparação de metodologia de regionalização de vazões. Geociência, v. 33, n. 3, p. 506-515, 2014.

NAGHETTINI, M. \& PINTO, E.J.A. Hidrologia Estatística. Belo Horizonte: CPRM, 2007. 552 p.

OLIVEIRA, P.T. Estudo estatístico sobre eventos de precipitação intensa no Nordeste do Brasil. 2014. 114 p. Tese (Doutorado em Ciências Climáticas) - Centro de Ciências Exatas da Terra, Universidade Federal do Rio Grande do Norte, Natal.

REBOITA, M.S.; GAN, M.A.; ROCHA, R.P.; AMBRIZZI, T. Regimes de Precipitação na América do Sul: Uma Revisão Bibliográfica. Revista Brasileira de Meteorologia, v. 25, p. 185-204, 2010.
RIBEIRO JÚNIOR, P.J. \& DIGGLE, P.P. geoR: A Package for Geostatistical Analysis. R - News, v. 21, n. 2, p. 15-18, 2001.

RICHARDS, J.A. Remote Sensing Digital Image Analysis: An Introduction, Second Edition, Springer-Verlag, Berlin, 1993.

SATYAMURTY, P.; NOBRE, C.A.; SILVA DIAS, P.L. Tropics - South America. In: KAROLY, D.J.; VICENT. D.G. (Org.). Meteorology of the Southern Hemisphere, v. 27, n. 49, p. 119-139, 1998.

SOUZA, A.; FERNANDES, W.A.; ALBREZ, E.A.; GALVÍNCIO, J.D. Análise de Agrupamento da Precipitação e da Temperatura no Mato Grosso do Sul. ACTA Geográfica, v. 6, n. 12, p. 109-124, 2012.

TUCCI, C.E.M. Regionalização de vazão. Porto Alegre: UFRGS/ABRH, 2002, 256p.

TUCCI, C. E. M. Hidrologia: ciência e aplicação. Porto Alegre: UFRGS/ABRH, 2007. 943 p.

VIOLA, M.R.; MELLO, C.R.; PINTO, D.B.F.; MELLO, J.M.; ÁVILA, L.F. Método de interpolação espacial para o mapeamento da precipitação pluvial. Revista Brasileira de Engenharia Agrícola e Ambiental, v. 14, n. 9, p. 970-978, 2010.

WILKS, D.S. Statistical Methods in the Atmospheric Sciences. 2. Filadélfia: Elsevier Academic Press Publications, 2006. 634 p.

XAVIER, T.M.B. S. \& XAVIER, A.F.S. Classificação e monitoração de períodos secos ou chuvosos e cálculo e índices pluviométricos para região Nordeste do Brasil. Revista Brasileira de Engenharia/Caderno de Recursos Hídricos, v. 5, n. 2, p. 7-3, 1987. 